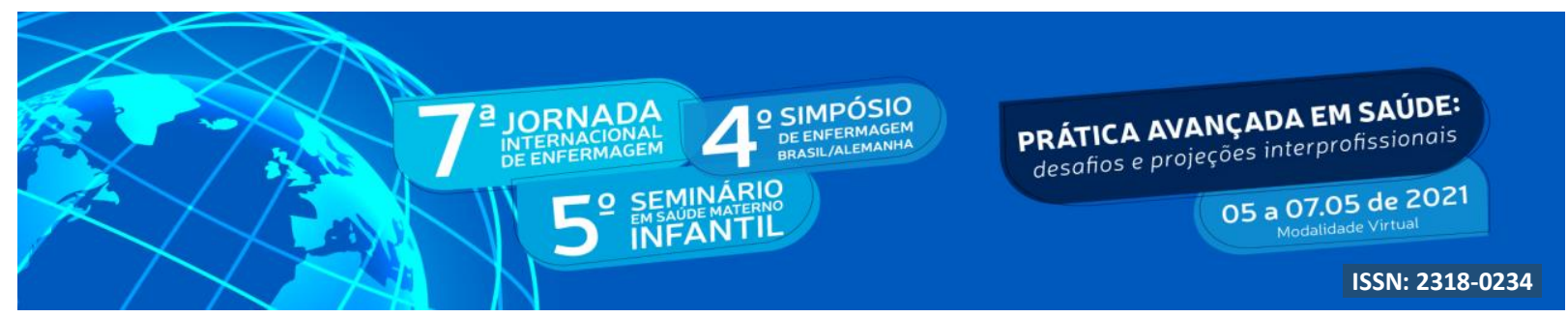

DOI: http://doi.org/10.48195/jie2021-030

\title{
ÓBITOS MATERNOS SEGUNDO CAUSAS DIRETAS E INDIRETAS: UM ESTUDO ECOLÓGICO
}

\author{
Marla Ariana Silva'; Rayssa Nogueira Rodrigues²
}

\begin{abstract}
RESUMO
Descrever os óbitos maternos segundo causas diretas e indiretas no estado de Minas Gerais e Brasil, período 2000 a 2018. Trata-se de um estudo ecológico, descritivo realizado no estado de Minas Gerais e Brasil. As informações de mortalidade materna, foram retiradas do painel de monitoramento da mortalidade materna, disponibilizadas pela Secretária de Vigilância em Saúde. No período de 2000 a 2018 verificou-se que a causa obstétrica direta foi a mais prevalente, sendo registrados $84 \%(n=1.129)$ óbitos em Minas Gerais e 72\% (n=14.348) óbitos no Brasil. Quanto às causas obstétricas indiretas, foram registrados $16 \%(n=146)$ óbitos em Minas Gerais e $28 \%(n=3.005)$ no Brasil. Os achados dessa pesquisa enfatizam a necessidade de maior investimento em estratégias implementadas pelo Ministério da Saúde.
\end{abstract}

Palavras-chave: Medidas em epidemiologia; Mortalidade materna; Saúde da mulher.

\begin{abstract}
Describe maternal deaths according to direct and indirect causes in the state of Minas Gerais and Brazil, from 2000 to 2018. This is an ecological, descriptive study carried out in the state of Minas Gerais and Brazil. The maternal mortality information was taken from the maternal mortality monitoring panel, made available by the Health Surveillance Secretary. In the period from 2000 to 2018 it was found that the direct obstetric cause was the most prevalent, with $84 \%(\mathrm{n}=1,129)$ deaths in Minas Gerais and 72\% $(n=14,348)$ deaths in Brazil. As for indirect obstetric causes, $16 \%(n=146)$ deaths were registered in Minas Gerais and 28\% $(\mathrm{n}=3,005)$ in Brazil. The findings of this research emphasize the need for greater investment in strategies implemented by the Ministry of Health.
\end{abstract}

Key Words: Epidemiology measures; Maternal mortality; Women's health.

\footnotetext{
${ }^{1}$ Enfermeira. Mestranda em Ciências pela Universidade Federal de São João del-Rei, Campus Centro Oeste Dona Lindu (UFSJ/CCO). E-mail: marla_ariana@hotmail.com.

${ }^{2}$ Enfermeira. Doutora em Enfermagem pela Universidade Federal de Minas Gerais (UFMG). Professor Adjunto I do Departamento de Medicina e Enfermagem da Universidade Federal de Viçosa (UFV). E-mail: rayssa_nr@yahoo.com.br.
} 


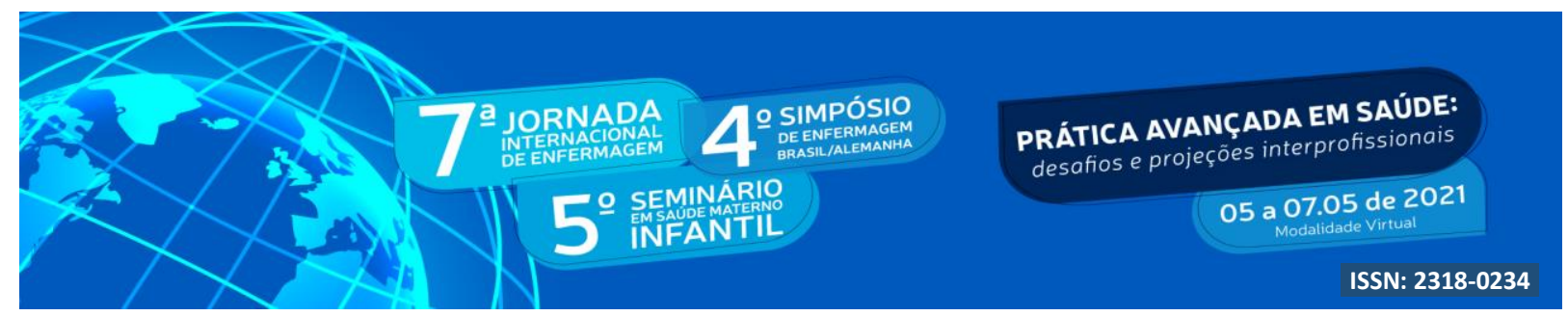

\section{INTRODUÇÃ̃O}

Conforme a Classificação Estatística Internacional de Doenças e Problemas Relacionados à Saúde (CID-10) e a Organização Mundial de Saúde (OMS), mortalidade materna é conceituada como:

A morte de uma mulher durante ou até 42 dias após o término da gravidez, independentemente da duração e local da gravidez, por qualquer causa relacionada ou agravada pela gravidez ou a sua gestão, mas não devido a causas acidentais ou incidentais (OMS, 1996).

Em relação à classificação, o óbito materno divide-se em obstétrico direto ou indireto. O primeiro resulta de complicações provenientes da gravidez, parto ou puerpério, como as síndromes hipertensivas e infecções puerperais (LIMA et al., 2016). Já o segundo ocorre quando há uma doença anterior à gestação ou que surgiu durante a gravidez e se agravou pela mesma, como as cardiopatias, diabetes, doenças sexualmente transmissíveis, entre outras (MEDEIROS et al., 2018). Além disso, existem também os fatores de risco socioeconômicos como nível de escolaridade, ocupação, ausência de companheiro e outras condições como idade materna, histórico prévio de saúde e número de realização de consultas de pré-natal (CARVALHO et al., 2015).

É indispensável destacar que quase todas as causas diretas são passíveis de prevenção. Quanto às causas indiretas, é relevante ressaltar que estão atreladas às mulheres já portadoras de doenças e devem, portanto, ser avaliadas, de início, como gestantes de risco e acompanhadas com mais cuidados (DIAS et al., 2015).

A mortalidade materna persiste como um grave problema de saúde pública (MARTINS; SILVA, 2018). Atualmente, mais de 303 mil mulheres no mundo continuam a morrer por ano devido a causas relacionadas ao ciclo gravídico puerperal (OPAS, 2018). Embora o Brasil seja um país de dimensões continentais, em contexto geral, nos últimos trinta anos, a hipertensão, a hemorragia, as infecções puerperais, as doenças do aparelho circulatório, complicadas pelo período gravídico-puerperal e o aborto foram as principais causas de morte materna em todo território nacional (MORSE et al., 2011).

Revela-se que, no intento de diminuir desigualdades e aperfeiçoar o Índice de Desenvolvimento Humano (IDH), o Brasil assumiu o pacto mediante oito iniciativas identificadas como "Objetivos de Desenvolvimento do Milênio (ODM)" entre as quais 


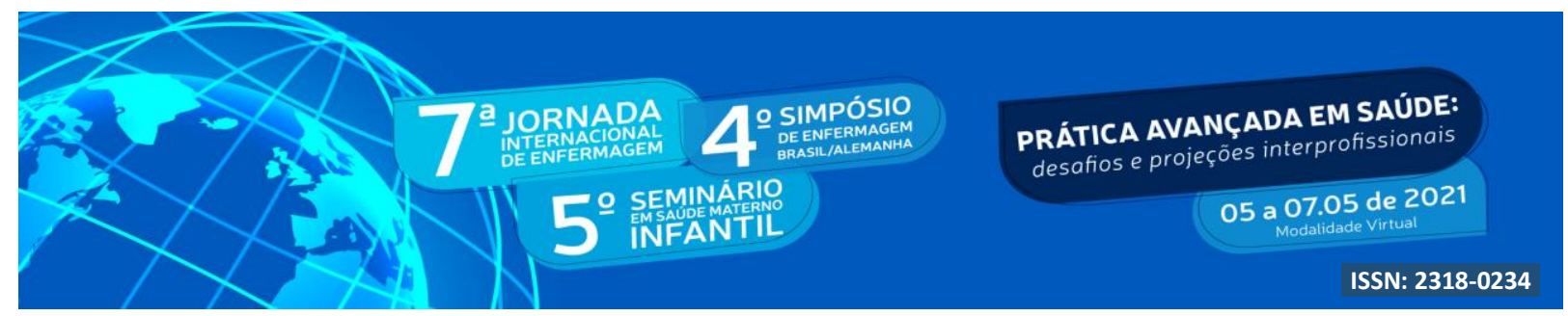

enfatizar-se a redução da mortalidade materna. Ressalva-se que o país é apontado pela OMS como um dos onze países latino-americanos que conquistaram aumentos expressivos na diminuição de óbitos relacionados à gravidez ou parto no período de 1990 a 2015 (GUIMARÃES et al., 2018).

Nesse contexto o Ministério da Saúde tem adotado, ao longo dos anos, políticas que propendam à melhoria da saúde da mulher. Entre determinadas pode-se destacar o Projeto Maternidade Segura de 1996; o Programa de Humanização do Pré-Natal e Nascimento (PHPN) de 2000; o Pacto Nacional pela Redução da Mortalidade Materna e Neonatal de 2002; A Política Nacional de Atenção Integral a Saúde da Mulher (PAISM) de 2003; A Lei do Acompanhante de 2005; e o destaque para a Rede Cegonha de 2011. Esses programas e políticas apresentam à tona a obrigação de que seja analisada assistência à saúde da mulher em todo o seu período de vida, e causam o enfermeiro como fundamental aliado na prevenção e diminuição da mortalidade materna (NARCHI; CRUZ; GOLÇALVES, 2013).

É imperativo salientar que grande parte das mortes maternas poderiam ser evitadas, com tecnologias leves presente nos dias atuais, especialmente se associadas aos avanços tecnológicos que são capazes de corrigir eventualidades. Neste sentido, procedimentos de baixo custo como: propedêuticas empregadas corretamente; atividades educativo-instrutivas; análise e coleta fidedigna da história pregressa e verificação minuciosa dos fatores de risco, são capazes de detectar parte dos problemas relacionados a mortes obstétricas, como exemplo às doenças hipertensivas específicas da gravidez (DHEG) que podem ser identificadas em consultas pré-natais na atenção básica (CARVALHO et al., 2014).

O trabalho de revisão realizado por Morse et al. (2011), o qual buscou publicações sobre a mortalidade materna disponibilizadas entre os anos de 1980 a 2010 revelou que existe um descompasso entre a importância dessa temática para a saúde pública e a quantidade de artigos produzidos sobre este assunto. Os resultados demonstraram que grande parte dos estudos estão concentrados na Região Sudeste em contrapartida existe uma baixa produção de informações acerca da realidade na região Norte, Nordeste e Centro-Oeste.

Diante do exposto, o estudo proposto poderá proporcionar o enriquecimento da comunidade acadêmica, com o aumento da produção científica na temática estudada, uma vez que há escassez de produções. Também será importante para despertar a necessidade de uma 


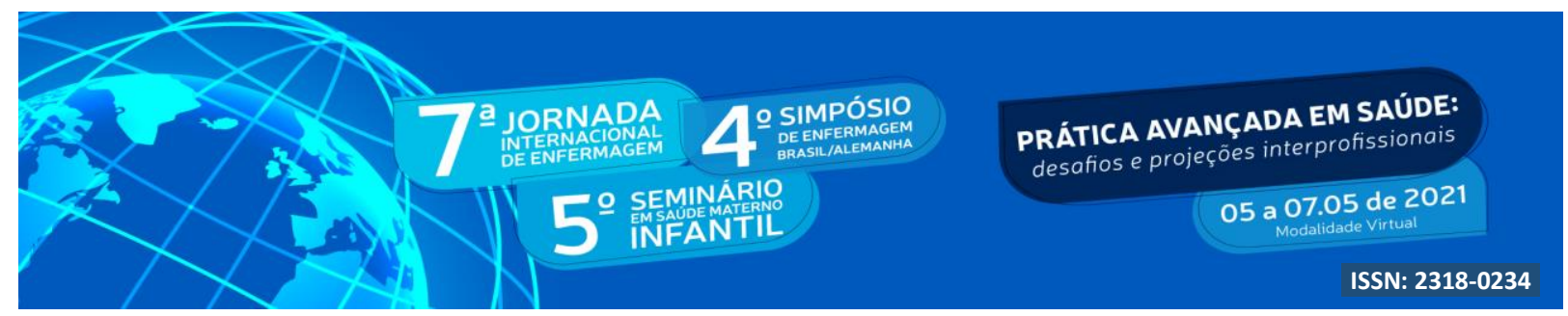

análise mais crítica sobre a assistência ofertada às mulheres, que poderia estar voltada para evitar ou reduzir as taxas de mortalidade materna.

\section{OBJETIVO}

Descrever os óbitos maternos segundo causas diretas e indiretas no estado de Minas Gerais e Brasil, período 2000 a 2018.

\section{METODOLOGIA}

Trata-se de um estudo ecológico, descritivo, realizado no estado de Minas Gerais e Brasil, período de 2000 a 2018.

O estado de Minas Gerais é o quarto maior em área territorial do Brasil e o segundo maior em número de habitantes, com uma população total estimada de 20.997.560 (IBGE, 2019). Já o Brasil, é o quinto país mais populoso do mundo e o maior da América Latina em área territorial (CENTRAL INTELLIGENCE AGENCY, 2017), possui uma população estimada de 210 milhões de pessoas (IBGE, 2019).

Os dados secundários, foram retirados do painel de monitoramento da mortalidade materna. Quanto a população-alvo deste estudo, consistiu nas mulheres residentes no estado de Minas Gerais e Brasil, registradas no Sistema de informações sobre mortalidade (SIM) por meio da Declaração de Óbito (DO).

Foram analisadas as seguintes variáveis: 1- Número de óbitos maternos declarados com causas obstétricas diretas (aborto, hemorragia, hipertensão, infecção); 2- Número de óbitos maternos declarados com causas obstétricas indiretas (Síndrome da Imunodeficiência Adquirida - Aids e doenças do aparelho circulatório complicando a gravidez, o parto e o puerpério).

Os dados coletados foram armazenados em um banco de dados eletrônico construído a partir Software Microsoft Office Excel (2016), posteriormente descritos em gráficos. Os resultados foram analisados utilizando estatística descritiva.

Por se tratar de um estudo em base de dados secundários, sua apreciação pelo Comitê de Ética em Pesquisa foi dispensada. 


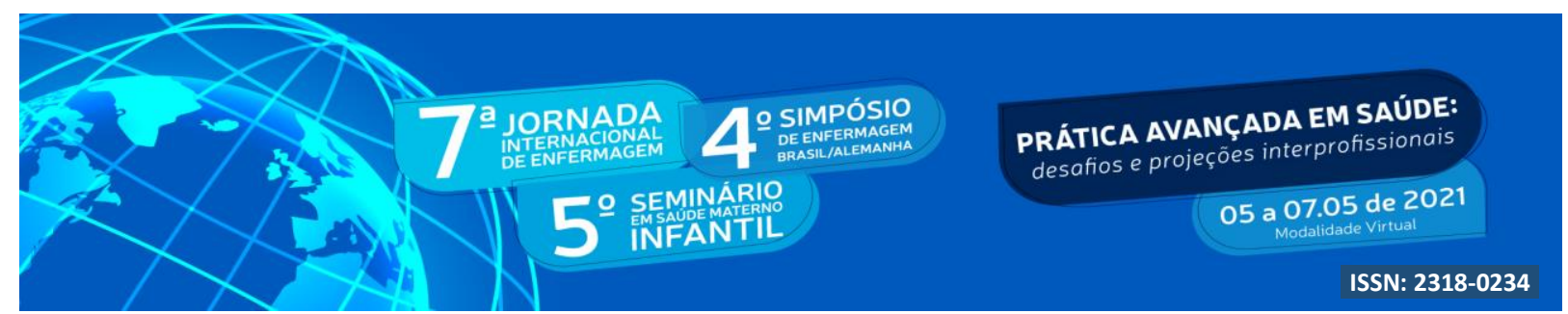

\section{RESULTADOS E DISCUSSÃO}

Entre o período de 2000 a 2018, verificou-se que a causa obstétrica direta foi a mais prevalente, sendo registrados 1.703 (84\%) óbitos em Minas Gerais e 21.983 (72\%) óbitos no Brasil. Quanto às causas obstétricas indiretas, foram registrados 335 (16\%) óbitos em Minas Gerais e 8.538 (28\%) no Brasil.

Quanto ao comportamento temporal, observa-se na Figura 1 que em Minas Gerais a mortalidade por causas obstétricas diretas e indiretas a hipertensão apresentou valores mais altos durante todo o período. Na Figura 2 o óbito materno por causas obstétricas diretas e indiretas para o Brasil também foi por hipertensão.

Figura 1: Óbito materno por causas obstétricas diretas e indiretas em Minas Gerais

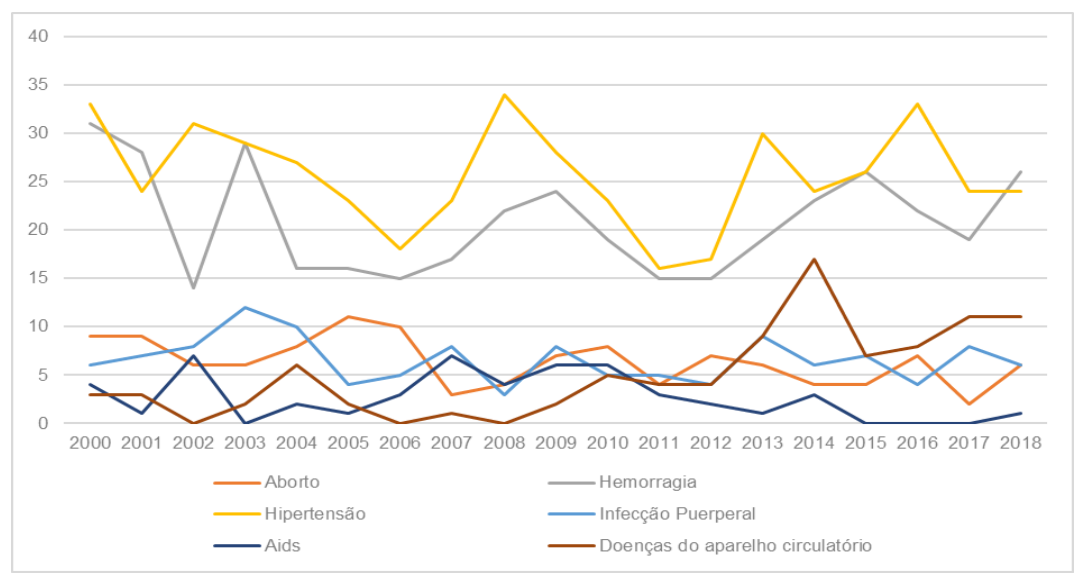

Figura 2: Óbito materno por causas obstétricas diretas e indiretas no Brasil

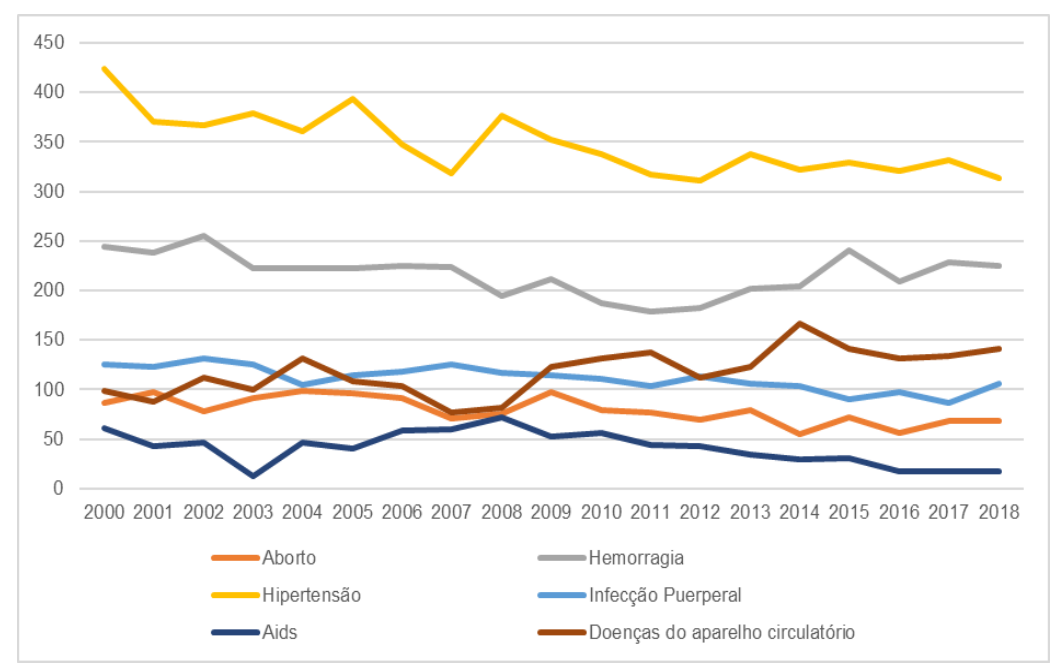




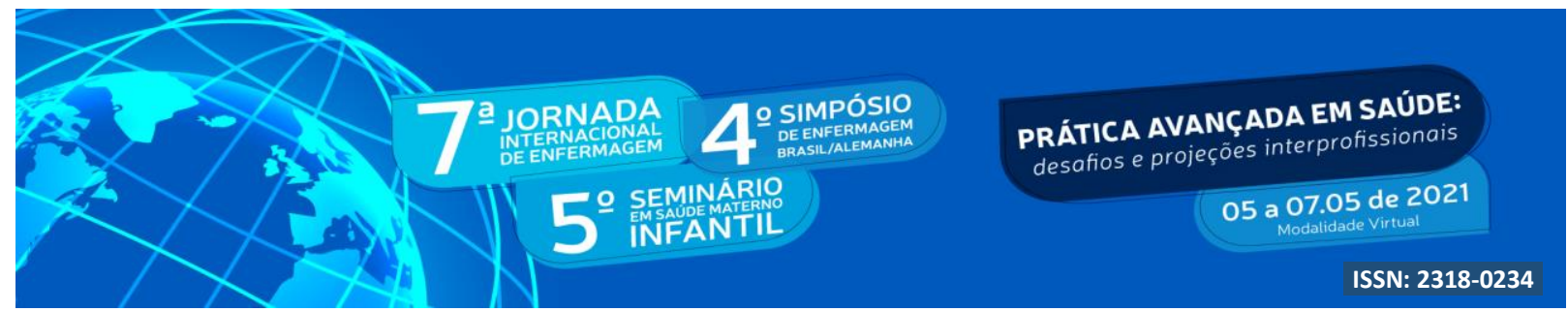

Entende-se por morte materna obstétrica direta aquela que ocorre por complicações obstétricas durante gravidez, parto ou puerpério, devido a intervenções, omissões, tratamento incorreto ou a uma cadeia de eventos resultantes de qualquer dessas causas; e morte materna obstétrica indireta aquela resultante de doenças que existiam antes da gestação ou que se desenvolveram durante esse período, agravadas pelos efeitos fisiológicos da gravidez (MEDEIROS et al., 2018). É importante destacar que quase todas as causas diretas são passíveis de prevenção (MARTINS; SILVA, 2018).

Destaca-se que as causas diretas ainda continuam sendo a principal causa de óbitos maternos. Dados internacionais apontam que mortes obstétricas diretas, causadas principalmente por hemorragia, aborto, doenças hipertensivas, gestação ectópica, ruptura uterina e sepse por infecção do trato genital ainda prevalecem. Entretanto pesquisa sobre a evolução e os desafios para o quinto objetivo de desenvolvimento do milênio, conclui que as causa indiretas estão ganhando importância notável nos países desenvolvidos (CARVALHO et al., 2014).

Neste estudo, a morte materna obstétrica direta representou o maior número de casos tanto em Minas Gerais quanto no Brasil, se comparadas a morte materna obstétrica indireta. Estudos realizados em Ribeirão Preto (FERNANDES et al., 2015) e na Bahia confirmaram esse resultado, mostrando a predominância de altas taxas para óbitos por causas obstétricas diretas (COELHO et al., 2016).

As causas obstétricas diretas que mais predominaram foi a hipertensão, presente em 43\% ( $n=487)$ em Minas Gerais e 46\% ( $n=6.608)$ no Brasil, hemorragia 35\% ( $n=396)$ em Minas Gerais e 29\% ( $n=4.118)$ no Brasil e infecção puerperal 11\% (n=125) em Minas Gerais e 15\% ( $\mathrm{n}=2.114)$ no Brasil. Resultado semelhante foi encontrado em um estudo realizado em Aracajú (BORGES et al., 2013).

No que tange as causas obstétricas indiretas, sabe-se que em algumas regiões, há a possibilidade das causas obstétricas indiretas não serem reconhecidas pela dificuldade do diagnóstico, pelo fato da paciente não informar doenças prévias ou até mesmo pelo próprio desconhecimento (VIANA; NOVAES; CALDERON, 2011). É importante observar que estão ligadas às mulheres já portadoras de doenças e devem, portanto, ser consideradas, de início, como gestantes de risco e acompanhadas com mais cuidados (MARTINS; SILVA, 2018). 


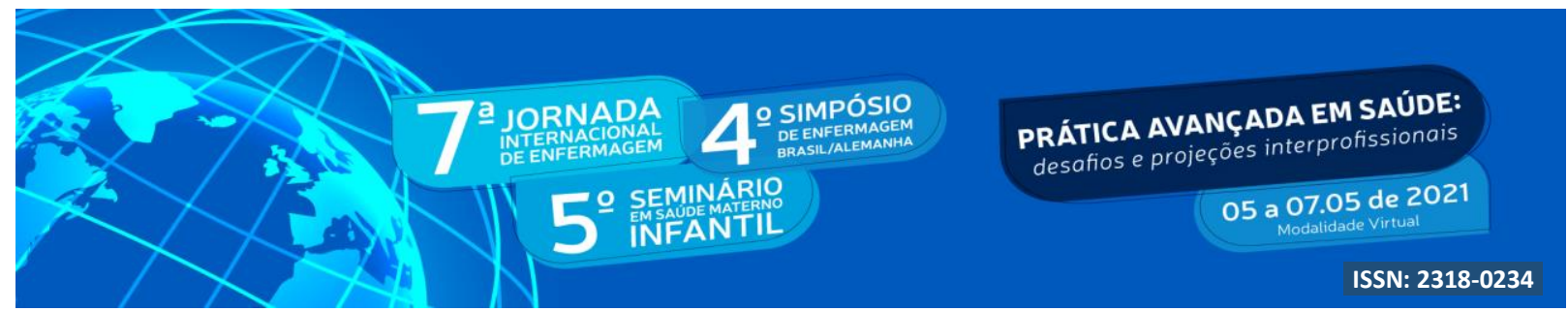

Entre as causas indiretas, o grupo com maior frequência foi o das doenças do aparelho circulatório complicadas pela gestação, pelo parto ou pelo puerpério, que representaram $65 \%$ $(n=95)$ em Minas Gerais e 74\% $(n=2.244)$ no Brasil de óbitos maternos. Corroborando com essa pesquisa, em São Luís (Maranhão), 35,4\% dos óbitos maternos corresponderam as doenças do aparelho circulatório (SILVA et al., 2016).

Limitações foram encontradas nesse estudo, pois as informações secundárias são provenientes dos sistemas de banco de dados já existentes. Nesse caso, os dados são de fontes nas quais não se pode garantir a ausência de erros e equívocos, tanto nos preenchimentos das fichas de notificação, como na digitação dos dados das fichas de notificação para as bases do painel de monitoramento. Outra limitação foi que algumas variáveis não são completamente valorizadas pelos registradores, comprometendo significativamente a análise retrospectiva dessas. Deve-se fazer um trabalho de conscientização para a valorização dessas informações, sensibilizando quanto à importância epidemiológica e social dessas variáveis e oferecer constantemente aperfeiçoamentos.

\section{CONCLUSÃO}

Ao buscar publicações acadêmicas relacionadas à mortalidade materna, evidencia-se pouco interesse pela produção e pelas atualizações de novos dados sobre a temática, ressaltando-se a necessidade de incentivar novos trabalhos para futuras publicações.

A mortalidade materna ainda é um problema de saúde pública nos diversos países subdesenvolvidos e em desenvolvimento, inclusive no Brasil. O presente estudo contribui para a atualização epidemiológica dos óbitos ocorridos no estado de Minas Gerais e no Brasil, visando proporcionar ferramentas para a melhoria na assistência prestada em ambos os locais estudados.

\section{REFERÊNCIAS}

BORGES, Cristiani Ludmila Mendes Sousa et al. Evolução temporal e diferenciais intraurbanos da mortalidade materna em Aracaju, Sergipe, 2000-2010. Epidemiologia e Serviços de Saúde, v. 22, n. 2, p. 307-316, 2013. 


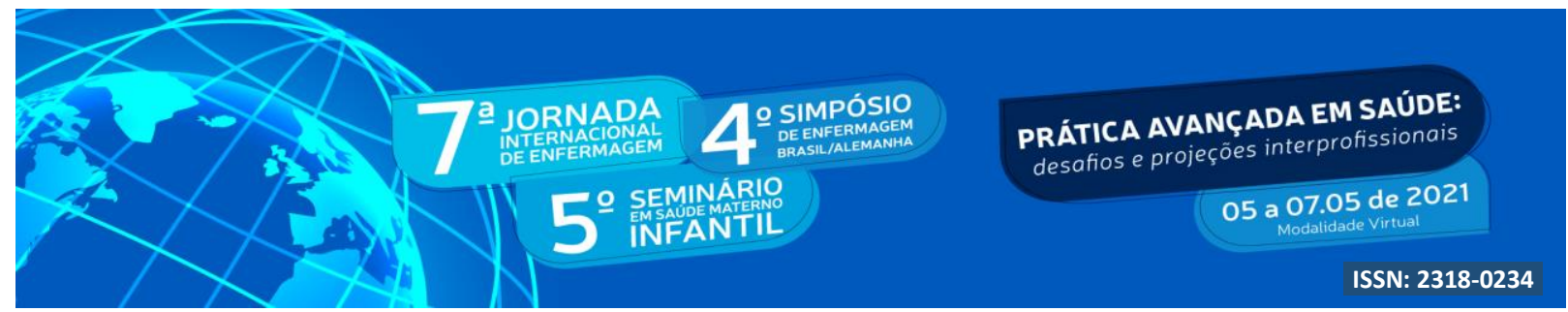

BRASIL. Ministério da Saúde. Secretaria de Atenção à Saúde, Departamento de Ações Programáticas Estratégicas. Manual dos Comitês de Mortalidade Materna. $3^{\mathrm{a}}$ ed. Brasília: Ministério da Saúde, 2007.

CARVALHO, Márcia Valéria Pereira et al. Mortalidade materna na capital do Piauí. Revista Interdisciplinar, v. 7, n. 3, p. 17-27, 2014.

CARVALHO, Moacira Lopes et al. Prevenção da mortalidade materna no pré-natal: uma revisão integrativa. Revista Interdisciplinar, v. 8, n. 2, p. 174-180, 2015.

CARVALHO, Patrícia Ismael de et al. Perfil sociodemográfico e assistencial da morte materna em Recife, 2006-2017: estudo descritivo. Epidemiologia e Serviços de Saúde, v. 29, p. e2019185, 2020.

CENTRAL INTELLIGENCE AGENCY. The World Factbook: Country comparison: area 2017. Washington, DC: CIA, 2017. Disponível em: https://www.cia.gov/Library/publications/the-world-factbook/rankorder/2147rank.html. Acesso em 7 jun. 2020.

COELHO, Vanessa Correia et al. Caracterização dos óbitos maternos em três regiões de saúde do centro-norte baiano. Cogitare Enfermagem, v. 21, n. 1, 2016.

DIAS, Júlia Maria Gonçalves et al. Mortalidade materna. Rev Med Minas Gerais, v. 25, n. 2, p. 173-179, 2015.

FERNANDES, Beatriz Boleta et al. Pesquisa epidemiológica dos óbitos maternos e o cumprimento do quinto objetivo de desenvolvimento do milênio. Revista Gaúcha de Enfermagem, v. 36, n. SPE, p. 192-199, 2015.

GUIMARÃES, Thaíse Almeida et al. Mortalidade materna no Brasil entre 2009 e 2013. Revista de Pesquisa em Saúde, v. 18, n. 2, 2018.

INSTITUTO BRASILEIRO DE GEOGRAFIA E ESTATÍSTICA (IBGE). População estimada, 2019. Disponível em: https://www.ibge.gov.br/cidades-e-estados/mg.html. Acesso em 29 de abr. 2020.

LIMA, Debora Rodrigues et al. Análise dos fatores intervenientes da mortalidade materna [Analysis of the intervening factors in maternal mortality]. Enfermagem Obstétrica, v. 3, p. e25, 2016.

MARTINS, Ana Claudia Sierra; SILVA, Lélia Souza. Perfil epidemiológico de mortalidade materna. Revista Brasileira de Enfermagem, v. 71, p. 677-683, 2018.

MEDEIROS, Lidiane Tavares et al. Mortalidade materna no estado do Amazonas: estudo epidemiológico. Revista Baiana de Enfermagem, v. 32, 2018. 


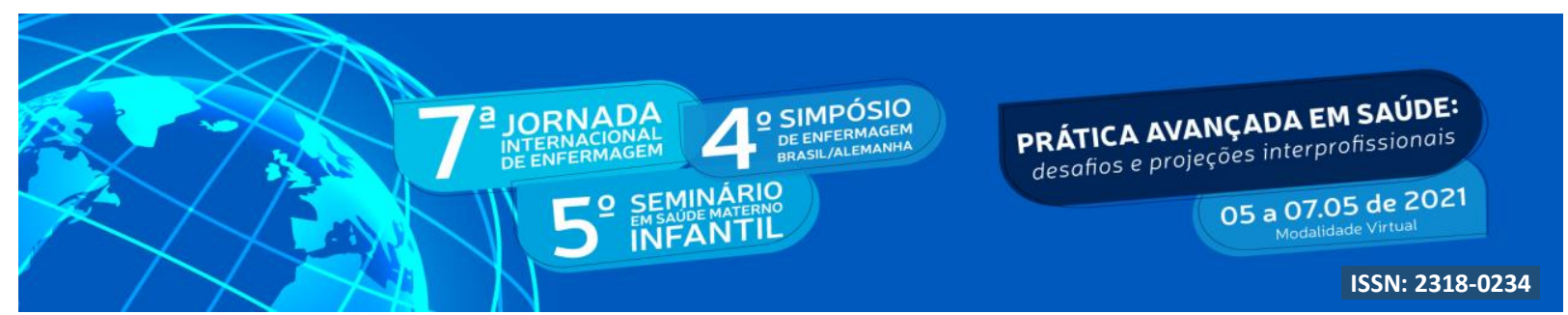

MORSE, Marcia Lait et al. Mortalidade materna no Brasil: o que mostra a produção científica nos últimos 30 anos?. Cadernos de Saúde Pública, v. 27, n. 4, p. 623-638, 2011.

NARCHI, Nádia Zanon; CRUZ, Elizabete Franco; GONÇALVES, Roselane. O papel das obstetrizes e enfermeiras obstetras na promoção da maternidade segura no Brasil. Ciência \& Saúde Coletiva, v. 18, p. 1059-1068, 2013.

ORGANIZAÇÃO MUNDIAL DA SAÚDE (OMS). Classificação estatística internacional de doenças e problemas relacionados à saúde - décima revisão. 3a Ed. São Paulo: Edusp; 1996.

\section{ORGANIZAÇÃO PAN AMERICANA DE SAÚDE (OPAS). Folha informativa - Mortalidade materna. 2018. Disponível em:} https://www.paho.org/bra/index.php?option=com_content\&view=article\&id=5741:folhainformativa-mortalidade-materna\&Itemid=820. Acesso em 27 abr. 2020.

SILVA, Bruna Gonçalves Cordeiro da et al. Mortalidade materna no Brasil no período de 2001 a 2012: tendência temporal e diferenças regionais. Revista Brasileira de Epidemiologia, v. 19, p. 484-493, 2016. 